\title{
FENOLES SOLUBLES TOTALES Y SU RELACION CON LA INHIBICION DE LA RIZOGENESIS EN ESTACAS DE NOTHOFAGUS PUMILIO (POEPP. ET ENDL.) KRASSER
}

\section{TOTAL PHENOL CONTENT AND ITS RELATIONS WITH RHIZOGENESIS IN CUTTINGS OF NOTHOFAGUS PUMILIO (POEPP. ET ENDL.) KRASSER}

\author{
Mirtha Latsague V. \& Janina Lara G. \\ Departamento de Ciencias Biológicas y Químicas, Universidad Católica de Temuco. Manuel Montt 56, Temuco, \\ Chile. E-mail: mlatsagu@uct.cl
}

\begin{abstract}
RESUMEN
Los compuestos fenólicos en concentraciones altas participan como inductores de la oxidación de auxinas, interfiriendo con la formación de raíces adventicias en estacas de tallo. Al no obtener enraizamiento en estacas de lenga (Nothofagus pumilio (Poepp. et Endl.) Krasser) en invierno y verano, se atribuyó que esto se debía a una alta cantidad de fenoles producidos en lenga, comparado con otros Nothofagus. En este estudio se procedió a cuantificar el contenido de fenoles solubles totales en estacas de lenga a través del método analítico Folin-Dennis, y comparar con el contenido de fenoles solubles totales en raulí (Nothofagus alpina (Poepp. et Endl.) Oerst.), especie que sí forma raíces adventicias. El contenido de fenoles solubles obtenidos en $N$. pumilio fue mayor $(2254 \pm 296 \mathrm{ppm})$ que en $N$. alpina $(1978 \pm 137$ ppm) $(\mathrm{P}<0.05)$. Los resultados coinciden con la propuesta que la no formación de raíces adventicias en estacas de lenga sería influida por altas concentraciones de fenoles, siendo necesario continuar con esta investigación en el sentido de identificar tipos de fenoles y comprobar in vitro el efecto de los fenoles como inductores de la actividad enzimática responsable de la oxidación de la auxina
\end{abstract}

Palabras claves: compuestos fenólicos, Nothofagus pumilio, rizogénesis.

\begin{abstract}
The failure to obtain rooting in cuttings of lenga (Nothofagus pumilio (Poepp. et Endl.) Krasser), has been attributed to higher phenol levels than those present in other Nothofagus. To test this idea, the Folin-Dennis analytical method was used to quantify the content of total soluble phenols in $N$. pumilio cuttings, and then compared to the phenols content in raulí (Nothofagus alpina (Poepp. et Endl.) Oerst.), a species that does form adventitious roots. Total soluble phenols in lenga were higher $(2254 \pm 296 \mathrm{ppm})$ than in raulí $(1978 \pm 137 \mathrm{ppm})(\mathrm{P}<0,05)$. The results support the proposal that relatively high phenol levels may be involved in the failure of adventitious rooting in N. pumilio cuttings. Further studies are needed to identify phenol types and to determine in vitro the effect of these phenols as inductors of enzyme activity responsible for auxin oxidation.
\end{abstract}

KeYwords: Nothofagus pumilio, phenolic compounds, rhizogenesis. 


\section{INTRODUCCION}

Existe información para un gran número de especies leñosas que responden al enraizamiento al ser tratadas con auxinas, entre ellas las del género Nothofagus. Es ampliamente conocido que el género Nothofagus responde al enraizamiento de estacas al ser tratadas con auxinas, particularmente AIB (Silva 1968; Santelices 1993, 1998).

No obstante lo anterior, Romero (2000) y Castro (2001) han demostrado que ello no ocurre con lenga (Nothofagus pumilio (Poepp. et Endl.) Krasser). Según los resultados obtenidos por estos autores, lenga no responde al tratamiento con AIB en ningún rango de concentraciones (500-4000 ppm). Se tomaron estacas de árboles de distintas procedencias, distintas edades (adultos y 5 años), distintas épocas (invierno y verano), sin llegar a la formación de raíces adventicias. Teóricamente esto se podría atribuir a la presencia de compuestos fenólicos (Phythoud \& Buchala 1989).

Las relaciones entre los fenoles y la rizogénesis inducida por la auxina pueden ser muy variadas, en algunas ocasiones actúan como cofactores rizogénicos, apoyando la fase de iniciación radicular y en otras dependiendo de la calidad y cantidad en que se encuentren pueden influir sobre la oxidación de la auxina (Zobel \& Talbert 1994), la cual está catalizada por la peroxidasa, impidiendo la formación de raíces adventicias. Muchos autores sugieren que AIA oxidasa es una peroxidasa, la cual, entre otras funciones celulares, controla el contenido endógeno de AIA (Phythoud \& Buchala 1989; Beffa et al. 1990; Rodríguez \& Tamés 1983; Hoyle 1972).

Frente a las dificultades de lenga de formar raíces adventicias a partir de esquejes y su posible relación con el contenido de fenoles, nos propusimos como objetivo cuantificar fenoles solubles totales en estacas de lenga utilizando la técnica de FolinDennis y comparar los resultados con el contenido de fenoles solubles totales en raulí (Nothofagus alpina (Poepp. et Endl.) Oerst.) especie de Nothofagus que sí forma raíces adventicias según la literatura.

\section{MATERIALES Y METODOS}

Se trabajó con estacas del año de crecimiento, tomadas de plantas madres de lenga y de raulí, que se encontraban en vivero, ambas de 5 años de edad. La zona basal de la estaca, dimensionada a una longitud de $10 \mathrm{~cm}$, se congeló en nitrógeno líquido, antes de ser triturada, molida, y homogeiza. Para la extracción de fenoles, de este homogenizado se tomaron $0,30 \mathrm{~g}$, al cual se le agregó $50 \mathrm{ml}$ de acetona acuosa al $70 \% \mathrm{v} / \mathrm{v}$ (para inhibir la interacción fenolproteína), agitando durante 2 horas en un agitador orbital (Giner-Chávez 1996). Los extractos fueron analizados inmediatamente, y se conservaron dosis como contra muestras en refrigeración y oscuridad.

Para el ensayo colorimétrico se utilizó el método Folin-Dennis (AOAC 1997), el cual determina la cantidad de fenoles solubles totales y que se basa en la reducción del ácido fosfomolíbdico por los fenoles, en un medio líquido alcalino. Para ello, a cada matraz aforado de $50 \mathrm{ml}$ con las respectivas muestras patrones y blancos se le añadieron $2,5 \mathrm{ml}$ de reactivo Folin, luego de 3 minutos se le agregó $5 \mathrm{ml}$ de carbonato de sodio al $20 \%$ (p/v), se agitó, se enraisó con agua destilada y homogeinizó.

Se utilizó espectrofotometría ultravioleta visible leyendo las muestras a $765 \mathrm{~nm}$. Los valores obtenidos fueron contrastados con una curva patrón de ácido tánico, tomado como sustancia de referencia.

Finalmente se realizó un análisis estadístico de los resultados obtenidos, con un análisis no paramétrico utilizando la prueba U de MannWhitney (Zar 1974).

\section{RESULTADOS Y DISCUSION}

En extracto de lenga se obtuvo un promedio de $2254 \mathrm{ppm}$ y para raulí un promedio de $1978 \mathrm{ppm}$ (Fig. 1). Los resultados obtenidos muestran diferencias significativas entre las cantidades de fenoles encontrados en estacas de las dos especies $(\mathrm{P}<0,05)$.

Es probable que la gran cantidad de fenoles presentes en lenga estén actuando como inductores de la oxidación de la auxina aplicada en el tratamiento, tal como lo informan Haissig (1974), Gaspar et al. (1982) y Marigo \& Boudet (1979). Estos autores señalan que la rizogénesis en la que AIA juega un rol fundamental, es también afectada por compuestos fenólicos, los que pueden modificar 
los niveles endógenos de AIA, e influir en el enraizamiento, por mecanismos que han sido relacionados con el metabolismo de auxinas.

Estudios realizados en plantas herbáceas como el maíz (Zea mays); demuestran que compuestos fenólicos como el 2-4 diclorofenol y el ácido cumárico fueron requeridos para catalizar la oxidación del AIA, aumentando la actividad de la enzima peroxidasa en las hojas de la plántula (Beffa et al. 1990) .

Lee et al. (1982) señalan que el ácido cumárico y la vainillina estimulan la oxidación de AIA. Por otro lado Phythoud \& Buchala (1989), trabajando con estacas de Populus tremula, han demostrado que se requiere monofenoles como cofactores para que peroxidasa pueda catalizar la oxidación de AIA. También se ha demostrado en tabaco (Sequeira \& Mineo 1966), rábano (Hoyle 1977) y tomate (Kokkinakis \& Brooks 1979) la actividad de la peroxidasa en este proceso de descarboxilación y su dependencia de los compuestos fenólicos. Gaspar (1981) señala, en un modelo de curva de actividad peroxidasa versus iniciación de raíces, que la cantidad de peroxidasa aumenta en la etapa de inducción y disminuye en la etapa de manifestación radicular, aumentando por tanto la actividad de la peroxidasa con los fenoles entre otros compuestos químicos.

Las plantas leñosas, en general, presentan grandes dificultades para expresar enraizamiento adventicio a partir de estacas. Esta característica podría deberse a que la competencia celular es baja, lo que impide la expresión morfogénica de los tejidos involucrados en el proceso. Lenga no escapa a estas características ya que tiene grandes dificultades para formar raíces adventicias a partir de esquejes, lo que se puede atribuir a la gran cantidad de fenoles solubles producidos, o a la calidad de éstos, los cuales estarían induciendo la oxidación de las auxinas aplicadas en los tratamientos y muerte celular en el lugar de corte.

Inicialmente se atribuyó este comportamiento de lenga a un severo receso metabólico invernal. Sin embargo esta hipótesis fue desestimada, por cuanto, que según estudios realizados en época de verano, con distintos tipos de estacas (apical, media y basal), tratadas en un rango de concentraciones de AIB (500-4000 ppm) y mantenidas durante 6 meses en invernadero, no se logró enraizamiento en ninguno de los tratamientos realizados, a pesar de haber probado con estacas de distintas procedencias y edades (Castro 2001).

En conclusión las altas concentraciones de fenoles solubles encontrados en lenga estarían induciendo la oxidación de la auxina AIB aplicada al tratamiento de enraizamiento. Aunque es probable que la formación de raíces adventicias en lenga no depende sólo de un factor, sino de un conjunto de factores, el estudio presente aporta evidencia que la cantidad de fenoles producidos probablemente sea uno de ellos. Se propone continuar con los estudios para poder identificar los fenoles y analizar in vitro el efecto de éstos sobre la actividad enzimática y así poder aportar mayores antecedentes sobre la inhibición de rizogénesis en estacas de lenga.

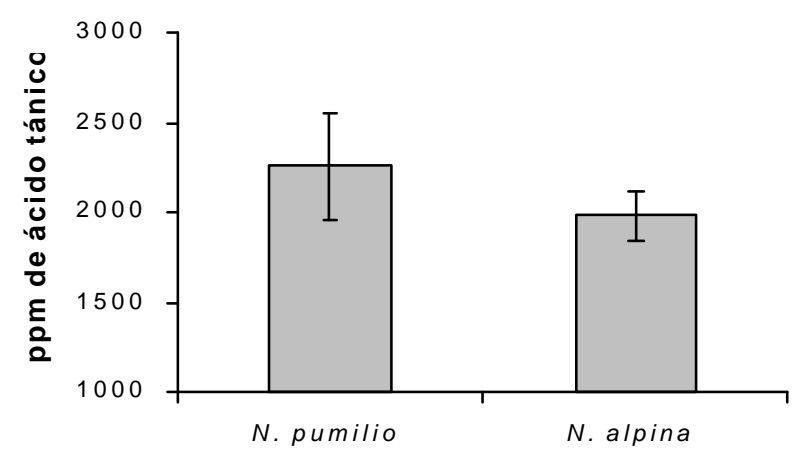

Figura 1: Promedios de índices de fenoles solubles totales en estacas de Nothofagus pumilio y Nothofagus alpina (expresados como ppm de ácido tánico) y sus respectivas desviaciones estándar. $(\mathrm{P}<0,05)$. 


\section{AGRADECIMIENTO}

Los autores agradecen a la Dirección de Investigación a través del Proyecto DIUCT 99-4-05.

\section{BIBLIOGRAFIA}

AOAC. 1997. Official Methods of Analysis of AOAC International. 16th edn. AOAC. International, Gaithersburg, Maryland, USA.

BefFa, R., H. Martin \& P. Pilet. 1990. In Vitro Oxidation of indol acetic acid by Soluble auxin-oxidases and peroxidases from maize roots. Plant Physiology 94: 485-491.

Castro, E. 2001. Propagación vegetativa de lenga (Nothofagus pumilio (Poepp. et. Endl.) Krasser) mediante enraizamiento de estacas de verano. Tesis Ingeniería Forestal, Universidad Católica de Temuco, Chile.

GASPAR, T. 1981. Rooting and flowering, two antagonistic phenomena from a hormonal point of view. En: Aspects and Prospects of Plant Growth Regulators. Monograph 6 (ed. B. Jeffcoat), British Plant Growth Regulator Group, Wantage, 39-49. pp. 39-49.

Gaspar, T., C. Penel, T. Thorpe \& H. Greppin. 1982. Peroxidases 1970-80. A survey of their Biochemical and Physiological Roles in Higher Plants. University of Geneva Press, Switzerland.

Giner-Chávez, B. 1996. Condensed tannins in tropical forages. $\mathrm{PhD}$ thesis, Cornell University, Ithaca, NY,USA.

HaIssig, B. 1974. Influences of auxins and auxin synergists on adventitious root primordium initiation and development. New Zealand Journal of Forestry Sciences 4: 311-323.

Hoyle, M. 1972. Indolacetic acid oxidase: a dual catalytic enzyme? Plant Physiology 50: 15-18.

Hoyle, M. C. 1977. High resolution of peroxidaseindolacetic acid oxidase isoenzymes from horseradish by isoelectric focusing. Plant Physiology 60: 787-793.
KoKkINAKIs, D. \& J. BRooks. 1979. Hydrogen peroxidemediated oxidation of indole-3 acetic acid by tomato peroxidase and molecular oxygen. Plant Physiology 64: 220-223.

Lee, T., A. Starkatt \& J. Jevnikar. 1982. Regulation of enzymic oxidation of indole- 3 acetic acid by phenols: structure-activity relationships. Phytochemistry 21: 517-523.

Marigo, G. \& A. Boudet. 1979. Effects on increase in levels of phenolics compounds on the auxin content and growth of Lycopersicon esculentum $\mathrm{Z}$. Pflanzenphysiologie 92: 33-38.

Moncousin, C.H. \& T.H. Gaspar. 1983. Peroxidase as a marker for rooting improvement of Cynara scolmus L. cultured in vitro. Biochemie und Physiologie der Pflanzen 178: 263-271.

Phythoud, F. \& A. Bruchala. 1989. Peroxidase activity and adventitious rooting in cuttings of Populus tremula. Plant Physiology and Biochemistry 27: 503-551.

Romero, M. 2000. Propagación vegetativa en lenga (Nothofagus pumilio (Poepp. et Endl.) Krasser) mediante enraizamiento de estacas. Tesis Ingeniería Forestal, Universidad Católica de Temuco.

SAntelices, R. 1993. Propagación vegetativa de raulí, roble y coigüe a partir de estacas. Ciencia e Investigación Forestal 7: 37-48.

Santelices, R. 1998. Propagación vegetativa del hualo (Nothofagus glauca (Phil.) Krasser) mediante estacas procedentes de rebrotes de tocón. Tesis de Magíster en Ciencias Forestales, Universidad de Chile, Chile.

Sequeira, I. \& I. Mineo. 1966. Partial purification and kineses of indolacetic acid oxidase from tobacco roots. Plant Physiology 41: 1200-1208.

SiLVA，J. 1968. Arraigamiento de estacas de raulí (Nothofagus alpina (Poepp. et Endl.) Oerst. Tesis de Ingeniería Forestal. Universidad de Chile, Chile.

Zobel, B. \& J. TAlbert. 1994. Técnicas de Mejoramiento Genético de Árboles Forestales. Editorial Limusa S.A., México. 545 pp.

ZAR, J. 1974. Biostatistical Analysis. Prentice-Hall Biological Sciences. 620 pp.

Recibido: 01.12.02

Aceptado: 26.04.03 